\title{
ON FIXED POINTS OF DYNAMICAL SYSTEMS
}

\author{
CEM TEZER
}

(Communicated by Kenneth R. Meyer)

Dedicated to Prof. M. Ikeda in the thirtieth year of his research and teaching in Turkey

ABstract. Two fixed points of a topological dynamical system are said to be of the same type if there exists a homeomorphic conjugacy of the system into itself sending the one fixed point into the other. The system will be said to be homogeneous if all its fixed points are of the same type. We introduce algebraic methods to investigate related questions for the shifts of expanding maps.

\section{INTRODUCTION AND STATEMENT OF RESULTS}

Given a topological dynamical system $(\Sigma, T)$ fixed points $\xi_{0}, \xi_{0}^{\prime} \in \Sigma$ will be said to be of the same type if there exists a homeomorphism

$$
f: \Sigma \rightarrow \Sigma
$$

such that

$$
f \circ T=T \circ f
$$

and

$$
f\left(\xi_{0}\right)=\xi_{0}^{\prime}
$$

Clearly, being of the same type is an equivalence relation among fixed points. A dynamical system will be said to be homogeneous if all its fixed points are of the same type.

The study of properties of fixed points would be rendered uninteresting or void if the system in question has few or no fixed points. In particular, the concepts of type and homogeneity should be relevant for dynamical systems in which fixed points occur abundantly rather in the sense of the concluding remarks of $\S 4$ in [5]. In this respect, the present article can be understood as a sequel to [5]

Our purpose is to point out the natural artifacts that can be employed to study the fixed points and their types in, and the homogeneity of a dynamical

Received by the editors February 22, 1989 and, in revised form, July 20, 1989.

1980 Mathematics Subject Classification (1985 Revision). Primary 55M20, 54H20.

Key words and phrases. Expanding maps, Reidemeister numbers, zeta functions. 
system $\left(\Sigma_{a}, \sigma_{a}\right)$ defined by

$$
\begin{aligned}
& \Sigma_{a}=\lim _{(}(X, a)=\left\{\left(x_{i}\right)_{i \in Z^{+}}, a x_{i+1}=x_{i}, i \in Z^{+}\right\} \\
& \sigma_{a}\left(\left(x_{i}\right)_{i \in Z^{+}}\right)=\left(x_{i+1}\right)_{i \in Z^{+}}
\end{aligned}
$$

where $a: X \rightarrow X$ is an expanding map of a smooth compact manifold $X$ [4]. We fix this notation. It is known that an expanding map always has a fixed point. [4, Theorem 4.I]. We shall let $x_{0} \in X$ denote an arbitrary but fixed fixed point of $a$. Clearly $\xi_{0}=\left(x_{0}\right)_{i \in Z^{+}}$is a fixed point of $\sigma_{a}$. Indeed, there is a natural one-to-one correspondence between the fixed points of $\sigma_{a}$ and those of $a$, since $\left(x_{i}^{\prime}\right)_{i \in Z^{+}}$is a fixed point of $\sigma_{a}$ iff $x_{i}^{\prime}=x_{0}^{\prime}$ for all $i \in Z^{+}$and $x_{0}^{\prime}$ is a fixed point of $a$.

For the presentation of our results we need some concepts from the classical homotopy theory of fixed points [2]: Given a group endomorphism $\alpha: G \rightarrow G$, elements $g, g^{\prime} \in G$ are said to be $\alpha$-equivalent iff there exists $x \in G$ such that $x g=g^{\prime} \alpha(x)$. The number $R(\alpha)$ of $\alpha$-equivalence classes in $G$ is called the Reidemeister Number of $\alpha$.

We prove

1.1. Proposition. If $a: X \rightarrow X$ is an expanding map of a compact smooth manifold, then the number of fixed points of a (equivalently that of $\sigma_{a}$ ) is equal to the Reidememeister number $R\left(a_{\#}\right)$ of

$$
a_{\#}: \pi_{1}\left(X, x_{0}\right) \rightarrow \pi_{1}\left(X, x_{0}\right) .
$$

Indeed, the set of fixed points of $a$ is in a natural one-to-one correspondence with the set of $a_{\#}$-equivalence classes in $\pi_{1}\left(X, x_{0}\right)$.

This is not at all an unexpected result in view of the strong geometry of expanding maps. However we have failed to find it in the literature.

Writing $\operatorname{Ad}[g]: G \rightarrow G$ for the inner automorphism induced by $g \in G$, defined by

$$
\operatorname{Ad}[g](x)=g x g^{-1}
$$

we formulate

1.2. Proposition. If $a: X \rightarrow X$ is an expanding map of a compact smooth manifold, then the types of fixed points of $\sigma_{a}$ are in one-to-one correspondence with the shift equivalence $([5,6,7])$ classes in the set

$$
\left\{\operatorname{Ad}[\langle\lambda\rangle] \circ a_{\#},\langle\lambda\rangle \in \pi_{1}\left(X, x_{0}\right)\right\}
$$

1.3. Corollary. If $a: X \rightarrow X$ is an expanding map of a smooth compact manifold, $\left(\Sigma_{a}, \sigma_{a}\right)$ is homogeneous iff all endomorphisms

$$
\operatorname{Ad}[\langle\lambda\rangle] \circ a_{\#}: \pi_{1}\left(X, x_{0}\right) \rightarrow \pi_{1}\left(X, x_{0}\right), \quad\langle\lambda\rangle \in \pi_{1}\left(X, x_{0}\right)
$$

are shift equivalent.

1.4. Proposition. If $a$ is an expanding map of the Klein bottle, then $\sigma_{a}$ is inhomogeneous. 
It can be readily seen that 1.3 is a direct consequence of 1.2 . We also note that 1.1 and 1.2 are nicely compatible in that, if $\langle\lambda\rangle,\langle\mu\rangle \in \pi_{1}\left(X, x_{0}\right)$ are $a_{\#}$-equivalent, then $\operatorname{Ad}[\langle\lambda\rangle] \circ a_{\#}$ and $\operatorname{Ad}[\langle\mu\rangle] \circ a_{\#}$ are shift equivalent. (Indeed, conjugate.)

\section{PROOFS}

2.1. Proof of 1.1. Let $\bar{X}$ be the simply connected covering space of $X, p: \bar{X} \rightarrow$ $X$ the corresponding covering projection. Let's take a point $\bar{x}_{0} \in \bar{X}$ with $p \bar{x}_{0}=x_{0}$ and let $\bar{a}: \bar{X} \rightarrow \bar{X}$ be the unique lifting of $a$ with the property $\bar{a}\left(\bar{x}_{0}\right)=\bar{x}_{0}$. There is an isomorphism $i$ of $\pi_{1}\left(X, x_{0}\right)$ onto $\Gamma$, the group of covering transformations of $p: \bar{X} \rightarrow X$. For each $\langle\lambda\rangle \in \pi_{1}\left(X, x_{0}\right)$ we can understand $i(\langle\lambda\rangle)$ to be the unique covering transformation sending $\bar{x}_{0}$ to $\bar{\lambda}(1)$ where $\bar{\lambda}$ is the unique lifting of $\lambda$ with $\bar{\lambda}(0)=\bar{x}_{0}$. The fact that $a: X \rightarrow X$ is an expanding map is independent of the Riemann metric on $X$ : So we can take any Riemann metric on $X$ and lift it to $\bar{X}$ via $p$ thus ensuring that $p$ is a local isometry, each $i(\langle\lambda\rangle)$ is an isometry, and finally each $i(\langle\lambda\rangle) \circ \bar{a}$ is an expanding diffeomorphism with a unique fixed point. Now, to each $\langle\lambda\rangle \in \pi_{1}\left(X, x_{0}\right)$ we let $x_{0}^{\prime}=p\left(\bar{x}_{0}^{\prime}\right)$ correspond where $\bar{x}_{0}^{\prime}$ is the unique fixed point of $i(\langle\lambda\rangle) \circ \bar{a}$. First we show that every fixed point of $a$ corresponds in this manner to some $\langle\lambda\rangle \in \pi_{1}\left(X, x_{0}\right)$ : If $x_{0}^{\prime}$ is a fixed point of $a$, let $\bar{a}^{\prime}$ be the unique lifting of $a$ with $\bar{a}^{\prime}\left(\bar{x}_{0}^{\prime}\right)=\bar{x}_{0}^{\prime}$ for some $\bar{x}_{0}^{\prime} \in p^{-1}\left(x_{0}^{\prime}\right)$. As $\bar{a}$ and $\bar{a}^{\prime}$ are liftings of the same map, they differ by a covering transformation. Consequently there exists $\langle\lambda\rangle \in \pi_{1}\left(X, x_{0}\right)$ such that $\bar{a}^{\prime}=i(\langle\lambda\rangle) \circ \bar{a}$. Secondly we show that if the fixed point $x_{0}^{\prime}$ corresponds to $\langle\lambda\rangle,\langle\mu\rangle \in \pi_{1}\left(X, x_{0}\right)$ then the latter are $a_{\#}$-equivalent: There exist $\bar{x}_{0}^{\prime}, \bar{x}_{0}^{\prime \prime} \in p^{-1}\left(x_{0}^{\prime}\right)$ such that

$$
\begin{aligned}
i(\langle\lambda\rangle) \circ \bar{a}\left(x_{0}^{\prime}\right) & =\bar{x}_{0}^{\prime}, \\
i(\langle\mu\rangle) \circ a\left(\bar{x}_{0}^{\prime \prime}\right) & =\bar{x}_{0}^{\prime \prime} .
\end{aligned}
$$

Consequently

$$
\begin{aligned}
& i\left(\langle\lambda\rangle^{-1}\right)\left(\bar{x}_{0}^{\prime}\right)=\bar{a}\left(\bar{x}_{0}^{\prime}\right), \\
& i\left(\langle\mu\rangle^{-1}\right)\left(\bar{x}_{0}^{\prime \prime}\right)=\bar{a}\left(\bar{x}_{0}^{\prime \prime}\right) .
\end{aligned}
$$

On the other hand, since $\bar{x}_{0}^{\prime}, \bar{x}_{0}^{\prime \prime} \in p^{-1}\left(x_{0}^{\prime}\right)$, there exists $\langle\omega\rangle \in \pi_{1}\left(X, x_{0}\right)$ such that

$$
\bar{x}_{0}^{\prime \prime}=i(\langle\omega\rangle)\left(\bar{x}_{0}^{\prime}\right)
$$

Hence

$$
\begin{aligned}
i\left(\langle\mu\rangle^{-1}\right) \circ i(\langle\omega\rangle)\left(\bar{x}_{0}^{\prime}\right) & =\bar{a}\left(i(\langle\omega\rangle)\left(\bar{x}_{0}^{\prime}\right)\right) \\
& =i\left(a_{\#}(\langle\omega\rangle)\right) \circ \bar{a}\left(\bar{x}_{0}\right),
\end{aligned}
$$

and

$$
i\left(\langle\mu\rangle^{-1}\langle\omega\rangle\right)\left(\bar{x}_{0}^{\prime}\right)=i\left(a_{\#}(\langle\omega\rangle)\langle\lambda\rangle^{-1}\right)\left(\bar{x}_{0}\right)
$$


Therefore

$$
\langle\mu\rangle^{-1}\langle\omega\rangle=a_{\#}(\langle\omega\rangle)\langle\lambda\rangle^{-1}
$$

and

$$
\langle\omega\rangle\langle\lambda\rangle=\langle\mu\rangle a_{\#}(\langle\omega\rangle) .
$$

2.2. Proof of 1.2. This is a simple consequence of Theorem 4.5 and Lemmas 5.6, 5.10 in [5]. The fundamental group of the Klein bottle ([5]) is of the form

$$
G=\left\langle A, B \mid A B=B A^{-1}\right\rangle
$$

and an expanding map $a$ of the Klein bottle is determined up to homotopy by three integers $p, q, r$ with $|p|,|r| \neq 0,1, r$ odd. Given $a=a(p, q, r)$, we have

$$
a_{\#}=\varphi(p, q, r): G \rightarrow G
$$

defined by

$$
\varphi(p, q, r):\left\{\begin{array}{l}
A \rightarrow A^{p} \\
B \rightarrow A^{q} B^{r}
\end{array}\right.
$$

2.3. Proof of 1.4. $\sigma_{a}$ is homogeneous iff $\operatorname{Ad}\left[A^{U} B^{V}\right] \circ \varphi(p, q, r)$ is shift equivalent to $\varphi(p, q, r)$ for any $U, V \in Z$. But

$$
\operatorname{Ad}\left[A^{U} B^{V}\right] \circ \varphi(p, q, r)=\varphi\left((-1)^{V} p,(-1)^{V} q+2 U, r\right)
$$

and in order for this map to be shift equivalent to $\varphi(p, q, r)$, it is necessary by 6.1 in [5] that $p=(-1)^{V} p$, which cannot be if $V$ is odd.

\section{A computational EXAMPLE}

We would like to show how the Reidemeister number can be employed to calculate the number of fixed points and eventually the Artin-Mazur zeta function of an expanding map of the Klein bottle: By 1.1 counting the fixed points of $a=a(p, q, r)$ is the same as counting the $\varphi(p, q, r)$-equivalence classes in $G=\left\langle A, B \mid A B=B A^{-1}\right\rangle$. Every element of $G$ can be uniquely expressed in the form $A^{g} B^{h}$ and the elements $A^{g} B^{h}, A^{g^{\prime}} B^{h^{\prime}}$ are $\varphi(p, q, r)$-equivalent iff there exist integers $x, y$ such that

$$
A^{x} B^{y} A^{g} B^{h}=A^{g^{\prime}} B^{h^{\prime}} \varphi(p, q, r)\left(A^{x} B^{y}\right),
$$

or

$$
A^{x+(-1)^{y} g} B^{y+h}=A^{g^{\prime}} B^{h^{\prime}}\left(A^{p}\right)^{x}\left(A^{q} B^{r}\right)^{y},
$$

or

$$
A^{x+(-1)^{y} g} B^{y+h}=A^{g^{\prime}} B^{h^{\prime}} A^{p x} A^{q \pi(y)} B^{r y}
$$

in view of $A B=B A^{-1}$, where $\pi$ is the unique parity preserving function from $Z$ onto $\{0,1\}$. Finally

$$
A^{x+(-1)^{y} g} B^{y+h}=A^{g^{\prime}+(-1)^{h^{\prime}}(p x+q \pi(y))} B^{h^{\prime}+r y} .
$$


Consequently $A^{g} B^{h}$ and $A^{g^{\prime}} B^{h^{\prime}}$ are in the same $\varphi(p, q, r)$-equivalence class iff there exist integers $x, y$ such that

$$
\begin{gathered}
x+(-1)^{y} g=g^{\prime}+(-1)^{h^{\prime}}(p x+q \pi(y)) \\
y+h=h^{\prime}+r y,
\end{gathered}
$$

or upon regrouping

$$
\begin{gathered}
(-1)^{y} g-g^{\prime}=\left((-1)^{h^{\prime}} p-1\right) x+q \pi(y) \\
h-h^{\prime}=(r-1) y .
\end{gathered}
$$

Note that $h \equiv h^{\prime}(\bmod 2)$ as $r$ is odd. Let

$$
Q=\left\{\begin{aligned}
(g, h) \in Z \times Z, 0 \leq h \leq 2|r-1|-1 & \\
, 0 \leq g \leq|p-1|-1 & \text { if } h \text { even } \\
, 0 \leq g \leq|p+1|-1 & \text { if } h \text { odd. }
\end{aligned}\right.
$$

A simple inspection reveals that the set $\left\{A^{g} B^{h},(g, h) \in Q\right\}$ contains exactly two representatives of each $\varphi(p, q, r)$-equivalence class, hence

$$
\begin{aligned}
R(\varphi(p, q, r)) & =\frac{1}{2} \#(Q) \\
& =\frac{1}{2}|r-1|(|p-1|+|p+1|)=|r-1|+p \mid
\end{aligned}
$$

remembering $r, p \neq 0,1,-1$. Since

$$
[\varphi(p, q, r)]^{n}=\varphi\left(p^{n}, q\left(1+p+\cdots+p^{n-1}\right), r^{n}\right)
$$

$$
\begin{aligned}
\zeta_{a(p, q, r)}(z) & =\exp \left[\sum_{m=1}^{\infty} \frac{1}{m}\left|r^{m}-1 \| p^{m}\right| z^{m}\right] \\
& =\frac{1-\operatorname{sgn}(r)|p| z}{1-|p r| z}
\end{aligned}
$$

\section{REMARKS}

An explicit calculation of the number of fixed point types for general $p, q, r$ appears to be difficult owing to the complications that attend the computational aspects of shift equivalence of $\varphi(p, q, r)$ as indicated in 6.1 of [5].

Disproportionate as it is to the modest scope and aims of this work, we would like to conclude by formulating a conjecture. First notice, that the proofs of $1.1,1.2,1.3$ can be easily modified to apply to any hyperbolic endomorphism of an infranilmanifold. This is a true extension of the above results in view of the recently established fact that expanding maps are infranilmanifold endomorphisms ([3]). This leads us to the

Conjecture. If $a: X \rightarrow X$ is a hyperbolic endomorphism of an infranilmanifold, then $\left(\Sigma_{a}, \sigma_{a}\right)$ is homogeneous iff $a$ is a nilmanifold endomorphism. 


\section{REFERENCES}

1. M. Artin and B. Mazur, On periodic points, Ann. of Math. 81 (1965), 82-99.

2. R. F. Brown, The Leftschetz fixed point theorem, Scott, Foresman and Co., Chicago, Illinois, 1971.

3. M. Gromow, Groups of polynomial growth and expanding maps, IHES Publ. Math. 51 (1981), 53-73.

4. M. Shub, Endomorphisms of compact differentiable manifolds, Amer. J. Math. 91 (1969), 175-199.

5. C. Tezer, The shift on the inverse limit of a covering projection, Israel J. Math. 59 (1987), 129-149.

6. R. F. Williams, Classification of subshift of the finite type, Ann. of Math. 98 (1973), 120-153.

7. $\ldots$, Classification of 1-dimensional attractors, Proc. Symp. Pure Math. 14 (1970), 341361.

Department of Mathematics, Middle East Technical University, Ankara, Turkey 